\title{
THE MECHANISM OF LARYNGEAL PITCH
}

\author{
LEON H. STRONG \\ Department of Anatomy, University of Michigan \\ TWO FIGURES
}

Theories of pitch production based upon an incomplete knowledge of the finer arehitecture of the larnyx have invaded the literature to such an extent that an interpretation of its mechanism gained by observation with precision instruments is warranted at the present time.

The ancient belief, that the width of the glottis governed the pitch of a laryngeal tone, held by Aristotle and by those who succeeded him for more than two milleniums, was revived and sponsored by no less a noted academician than Dodart as late as 1707. A quarter century later (1741) Ferrein pioneered the physiological processes of the larynx by ingenious and thorough experimental studies on freshly killed dogs, oxen and swine, and in one case, upon a human cadaver shortly after death. He proved that sound originated when and where the glottic edges, which he named cordes vocales, met and were parted by a pneumatic blast, and, in addition, that pitch was controlled by the contraction of the intrinsic musculature of the larynx. Close to a century later (1839), Johannes Müller repeated the experiments of Ferrein, confirmed them and added the observation that "The notes are, ceteris paribus, lower in pitch in proportion to the length of the glottis," thereby correcting Ferrein's almost single error, that the height of the tone was proportionate to the length of the vocal cord. In '31, Russell succeeded in making a kinetograph of a normal larynx in song, thereby displaying for the first time, certain phenomena which accompany phonation. 
The physiological processes brought to view by the kinctograph made imperative a reexamination of the finer structure of the larynx, which has demonstrated a mechanism adequate to render all modulations of pitch of which the organ is capable. This investigation has consisted of an analysis of those structures of the larynx which might act as a sound producing mechanism according to known physical facts; a checking of these facts against the observations of the mechanism as shown by the kinetograph; direct examination of the larynx in the living with the laryngoscope, upon persons capable of emitting normal tones while the observations were taking place; observations of roentgenographs of the Iarynges of singers producing specific rates of vibration; and making application of certain pathological phenomena in interpretation of the mechanism. These observations and a structural analysis of the larynx have led to an integrative interpretation of the physiological processes displayed by laryngeal mechanies, in the production of pitch.

From about the middle of the last century until 1887 there was a variety of opinions expressed in studies in the literature as to the structure of the large muscle mass called the thyroarytenoid muscle, which is one of the structures comprising the vocal lip. At this time Jacobson made a careful and extensive study of this muscle, a certain group of fibers of which had both been affirmed to be present and denied by certain of his predecessors. He showed that certain of the medial fibers of this muscle mass which he designated the aryvocalis were physiologically separate from the lateral fibers. The importance of the presence of such muscle fibers seems to have been little realized by those who have essayed to solve the problem of pitch production, from Jacobson's time to the present.

A significant aid to the present study was the invention by Russell of the laryngoperiscope, and, by means of this the production of a kinetograph of his own larynx during song. The study of this film allowed for the first time a clear view of just what happened in the larynx during intonation. The phenomena displayed were at variance in many respects with opinions, and even observations, expressed in the literature. 
The writer's observations are here recorded after having been afforded a study of the film by Doctor Russell, which permitted analyses of the various points of interest hypothecated by, and of interest to him. Since the kinetoscope could be stopped at will, and the kinetograph could be repeated as often as desired, a careful analysis was possible. Doctor Russell very kindly stood by and accompanied the film vocally as it was projected on the screen, thus giving essentially a talking picture effect. The writer wishes to absolve Doctor Russell from any interpretation which is herein expressed at variance with his own interpretation, and to state frankly that without the aid of this film he could not have come to the conclusion here given.

In approaching a solution to the problem of pitch control it is necessary to keep in mind the fact that most notes originating in the larynx have vibration rates between 100 and 1000 cycles per second. Such frequencies are rapid. They are of the order of frequency of the radio broadeast band, and the structure which emits them must of necessity be very tenuous if elongate, and very small in any case. The vibrating structure is not in a partial vacuum and, therefore, if large it would be hard to set into such rapid vibration with so small a force as the air pressure developed in the lungs. Moreover the mechanism is capable of changing vibration frequencies instantly over a great range of modulations. The degree of accuracy of pitch attained in passing from one part of the musical scale to a distant part suggests controlling mechanisms of micro-order. These facts indicated the necessity of looking to the finer gross and even histologic structure of the larynx rather than to its coarser details, for an analysis of its behavior. After a knowledge of the structure there remained need for a functional checking of some kind. Only indirect checking methods were available up to the time Russell made the motion picture of the larynx to which reference has been made. But, upon viewing this film it is possible to have a direct check upon the functions suggested by anatomical relationships. The processes shown are therefore easy of accurate interpretation. 
Some confusion concerning the glottic region has arisen because of the various definitions of the constitution of the so-called vocal cord. I shall adhere to the BNA terminology which appears to be perfectly clear. The following structures as defined will make clear the illustrations cited.

The vocal lip (labium vocale) is the glottic contact edge and the region immediately proximate to it (fig. 2). The vocal lip includes the vocal fold (plica vocalis) which is the mucous membrane only; the vocal ligament (ligamentum vocale) which is the upper thickened edge of the conus elasticus; the part of the conus proximate to the ligament; and that part of the thyroarytenoid muscle (m. thyroarytenoideus) designated the vocalis.

In the living, as seen by the laryngoscope, the vocal fold applied to the vocal ligament, is a glistening white of bluish cast. This white color indicates the non-vascular nature of these two structures which is in striking contrast to the ventricular fold above it and the rest of the visible larynx which, in color, is reddish. The vocal fold appears flat along its entire length. Russell succeeded in obtaining a sharply defined roentgenogram of the ventricle of a living female larynx. In this film the upper surface of the vocal fold appears to be a straight line, even when the subject was producing a note of 1024 cycles. We may assume then that the contraction of the muscle band (vocalis) which is in apposition with the fold did not take place in a dorsoventral direction during phonation, for if it contracted, a muscle as thick as the vocalis would show a rounded contour instead of a straight line. The only fibers of this muscle, then, which might contract and not show a rounded contour in the dorsoventral plane would be those whose force was oblique to the edge of the vocal lip and which were parallel to the ventricular surface of the vocal fold. These would be in the transvese plane of the body. Such a group of fibers of micro-order are those composing the aryvocalis part of the vocalis muscle.

Histologically the vocal fold is composed of two types of epithelium. It is stratified squamous over the glottic edge and 
for a few millimeters below it and laterally for about $3 \mathrm{~mm}$. into the floor of the laryngeal ventricle. At these limits the nature of the epithelium changes rather abruptly to tall columnar ciliated. The flat-celled epithelium shows clearly the abrasive character of the glottic edge, for it is the one area of the whole larynx with any continuous amount of contact epithelium. The aryvocalis muscle is separated from the air stream by a distance of 2 or $3 \mathrm{~mm}$. Between this muscle and the air stream are two structures, the vocal ligament and the
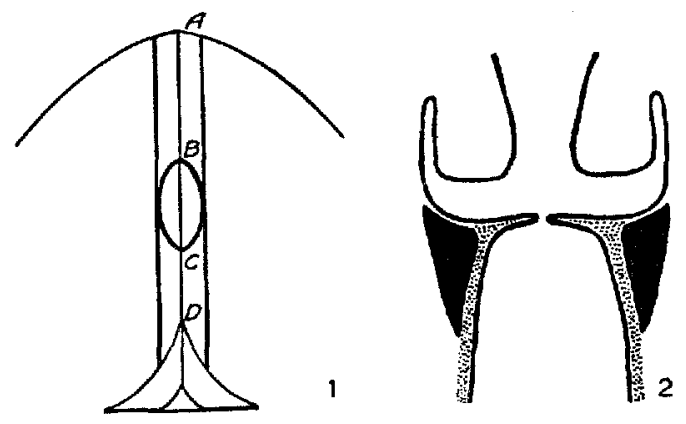

Fig. 1 Diagram of rosal lips and adnexa seen from above. The vocal lips are in contact in the midline. $A=$ lamina of thyroid cartilage. $B=$ ventral limit of vibrating segment. $\mathrm{C}=$ dorsal limit of vibrating segment. $\mathrm{D}=$ tip of voea! process of arytenoid cartilage. $\mathrm{AD}=$ vocal ligament covered by vocal fold. $\mathrm{AB}$ $=$ ventral non-vibrating segment. $\mathrm{BC} \ldots$ vibrating segment. $\mathrm{CD}=$ dorsal nonvibrating segment.

Fig. 2 Diagram of a coronal (frontal) section through the larynx showing the relation of the epithelium (black line), the conus elasticus and rocal ligament (stipple), and the thyroarytenoid muscle (solid black). The reduplication of the vocal fold is much exaggerated.

vocal fold. Since the ligament is comprehended between the laryngeal and ventricular surfaces of the fold and is bound to them intimately, the two almost form a single structure; a structure which is easily capable of vibration. Viewed from above, the ligament is covered by nothing but stratified squamous epithelium, but since a change in the type of epithelium occurs laterally where the muscle is in contact with the ligament, the muscle is covered by tall columnar ciliated epithelium. 
During song, the laryngoperiscope show the following facts. The segments of the edges of the vocal lip which are in contact partially or entirely, so that they impede the pneumatic assault from below, are longest for the lower tones, and shortest for the higher tones. Gradually as the pitch is raised the vibrating edges become shorter and shorter. Two configurations of the glottis occur in the production of ordinary tones. One, in which the whole of the diverging membranous glottic edges vibrates. A second, in which the membranous glottic edges are in contact throughout their entire length during vibration except for the vibrating area. This area consists of two small segments, one at each edge, equal in length and mutually opposed (fig. 1). The area appears to the eye oval in form, with the long axis dorsoventral. Those portions of the vocal lips which are not in contact during vibration appear to be a lax oval segment (half from each lip), which separates two tensed segments continuous with the oval segment at either end. The lax segment, a temporary valve, is the vibrating segment. When a falsetto tone in produced the single vibrating edges appear to assume suddenly the form of a double internodal or looped segment with a node between two loops, which fact recalls the appearance of the loops produced in physical experiments of vibrating strings. This appearance bears out that seen by Ortel in 1882 by means of the stroboscope in which the vibrating edges appeared in two sections. Upon resumption of the normal register, the double segment seems to snap into a single segment, the total length of the vibrating part of the vocal lip undergoing some increase. The vibrating edges are flooded constantly with mueus which appears in large and small droplets, filling the dorsoventral furrow indicating the closed glottis, formed by the glottic edges as they touch each other. 


\section{DISCUSSION}

Rise of pitch has been ascribed to the action of the cricothyroid muscle tensing the edge of the vocal lip by lengthening the dorsoventral diameter of the glottis in analogy to the tightening of a string of a stringed instrument. The vocal ligament as well as the conus elasticus is a structure composed almost entirely of elastic tissue. In its physical properties, therefore, it resembles somewhat a rubber band; that is, when tension is put upon it elongation takes place with proportionate diminution in ealiber so that tension does not produce a rise in pitch proportionate to the degree of tension. A rubber band may be increased to three times its unstretched length or more without altering its pitch. In this respect it differs strikingly from a steel string. The vocal ligament is somewhere between a steel string and a rubber band in its ability to alter its pitch with tension. The contracture of the cricothyroid muscle then could not alter the pitch of the tone emitted by the vocal ligament in such a ratio as the modulation known by experience requires. Since the only other means of stetching the vocal ligament is the up-tilting of the vocal process by the cricoarytenoideus posterior aided by the arytenoideus when the arytenoid cartilage shifts backward over the condylar surface of its articulation, and this is indeed less than $5 \mathrm{~mm}$., then some other means than tension of the vocal ligament must be found which could produce the great variety of pitches of which the voice is capable. Moreover, observations show that when a vibrating segment of the vocal lip is longer than one just before the lengthening has taken place, the note is lowered.

Muckey ('15) hypothecates an edge of the vocal lip with fibers extending into the vocal ligament of different lengths. So that with increasing tension on the vocal ligament less and less of the free lip could vibrate with a consequence that a lesser weight of vibrating structure would produce a higher pitch with the same amount of air pressure. Examination of of the muscle fibers clearly shows that they are not inserted as he postulates. Moreover, it would necessitate fibers of 
great strength pulling at an angle to the vocal ligament. What fibers of this direction which do exist are of micro-order, and their effect upon the vocal ligament can be but to tense feebly certain areas of the vocal ligament.

Negus ('29) asserts "that alterations in pitch are directly dependent on variation in degree of contraction of the thyroarytenoid muscles," and he derives the sound from the vibration of that muscle. He cites a clever experiment in which he stimulated two pieces of fresh frog muscle, in contact, and capable of being forced apart from below by a constant current of air to contract. He reports that the sound emitted by their edges rose in pitch proportionately to the intensity of the stimulus (electricity). Now this experiment has features which do resemble somewhat the vocal apparatus in producing sound, but the resemblance lacks identity. So the effect produced by the larynx can better be explained by an analysis of the facts rather than through analogies. When the thyroarytenoid muscle contracts it shortens, and pulls forward with it the arytenoid cartilage. The frog muscle above referred to did not shorten like the contracted thyroarytenoid. It was merely tensed and, of course, it would vibrate at a faster rate according to the physical laws of sound. It must be recalled, too, that the bulk of the thyroarytenoid muscle, or its peculiar wedge shape is not at all even similar to the gastrocnemius muscle of the frog which he used. In man the thyroarytenoid muscle, be it remembered, is separated from the blast of air by both the mucous membrane (vocal fold) and the vocal ligament, a distance of 2 or $3 \mathrm{~mm}$. And it is within the width of this distance of $3 \mathrm{~mm}$. that the whole of the vibrating segment appears, that is, vibration violent enough to be visible shows itself within a distance of the width of the vocal ligament.

The bulk of the contracted thyroarytenoid muscle is such that it would be an impediment to any vibration as rapid as that encountered in even the lowest human tones. It is to be emphasized that in ordinary song frequencies as high as 512 cycles occur. Whatsoever the vibrating structure be, it must be tenuous because the pneumatic assault from below, for 
low tones is only $12 \mathrm{~mm}$. of mercury, and for the very highest tones, which would be about 1024 cycles, it is only $73 \mathrm{~mm}$. of mercury. These are not great pressures. The bulk of the thyroarytenoid is physically too great to be set vibrating at from 500 to 1000 eycles by the amount of force which could be exerted against the valve made through the vocal lips by a pressure of approximately to $\frac{1}{2} \mathrm{gm}$., which is the approximate weight sustained by the valve, calculated for 5 sq.mm. area.

Comparative anatomical studies of the larynx suggest a factor in the evolution of the vibrating mechanism which is not to be overlooked in evaluating the roles of the structures aiding in pitch mechanics. In the lower forms (Amphibia) where first the glottis appears, the act of sound production is passive, the animal being in no way concerned with the sound about to be produced. Nor is there an auditory organ yet present to record pitch. The glottic margins are merely mutually approachable. Such a condition forms a valve for impeding the air. Elasticity of the valve permits rapid opening and closing of the glottic edges which initiates sound. If, as stated by some investigators the vibration of a tensed muscle causes sound there must have been a transition from the primitively passive condition to one which might be designated active. There is no good reason for so assuming. Such a transition from passive to active mechanisms is unnecessary because the vibrating structure is still passive. The nonvascular nature of the vibrating area (vocal fold and voeal ligament) of the vocal lips is an indication of its passive nature. Analysis of the vocal lip will show structures that are in such relation that variations in pitch may be attained without the vibration of any muscle at all, except as a secondary effect of a dissipated energy upon such a muscle.

Any physical structures which bear a certain relation to each other and which have properties to enable them to move rapidly may act as initiators of vibrations. Since the larynx consists of many and movable parts, vibrations may be initiated in more than one way. Because the aryvocalis fibers are 
set at an angle (as great as $45^{\circ}$, at times) to the vocal ligament and to the mass of the thyroarytenoid muscle which parallels the ligament, they exert a lateral pull upon the conus elasticus, especially upon its upper condensed edge, the vocal ligament. By specific innervation of any group of these fibers any segment or segments of the vocal ligament may be put under tension. This innervation is under continuous and instantaneous check by means of the acoustic apparatus. The vocal lips are approximated under mutual pressure laterally by the tonus of, and by the bulk of the thyroarytenoid musele aided by the structures lateral to it. The pressure exerted by the thyroarytenoid muscle mass, which acts as a strapped band on either side of the vocal lip, tends to keep the edges of the vocal lips in contact. These are relatively large muscles, whose principal function is to shut the glottis during deglutition, so that they exert no mean pressure medially against the conus elasticus. The aryvocalis fibers are of micro-dimensions and their pull laterally at an angle could not be sufficient to act in any way against the medial pressure of the thyroarytenoid mass. So that in essence these small aryvocalis fibers merely tense the edge of the conus and the vocal ligament whenever and wherever they are innervated. Wherever these fibers are not innervated along the edge of the vocal ligament, at any given moment, a section of this ligament is in a condition in which it will have less resistance to the pneumatic assault from below than the portions of the edge which are innervated. It is in this manner that the air can make its way through the glottic opening, which results from the denervated segments of the glottic edge being forced apart by the pneumatic blast. This is a wholly passive action. Once an opening is forced through the opposed glottic edges, then when the pressure is released by this opening the glottic edges spring back by elastic recoil and again block the passage of air. This rapid interruption of the air column becomes periodic, resulting in a definite pitch. Because the conus narrows toward the glottic edge rather abruptly, thus concentrating the unit area of force of the penumatic assault, little impedance will result in vibra- 
tion. This impedence need be very small for the vocal ligament is so elastic and tenuous.

If the segment guarding the glottic opening be extremely tenuous, great rapidity might be attained in opening and closing this valve, formed by each of the glottic edges, guarding symmetrically half the opening. A vibration of the approximated edges would result passively. If the segment vibrating were held under the same tension and the same bulk of vibratile edge were in use, then if the segment vibrating were increased in length the number of vibrations set up by a constant pressure of air would be decreased; contrariwise, if the segment vibrating were shortened the number of vibrations would be increased.

The stratified squamous epithelium of the vocal fold which is applied to the vocal ligament has a thickness of only four or five cells at the vibratile edge of the vocal lip. The vocal ligament fits medially into this fold, so that it forms there a very thin wedge of elastic connective tissue. This connective tissue increases in bulk laterally to form a surface 2 to 3 mm. in width which abuts against the medial surface of the thyroarytenoid muscle. This medial surface of the thyroarytenoid is that from which the aryvocalis fibers arise (as well as from the arytenoid cartilage) to pass ventromedially to an insertion into the vocal ligament.

Looking at figure 1 , we conceive the mechanism of the larynx to function, in the production of certain pitches, according to the following description. The vocal folds are approximated, closing the glottis. Segment of the vocal lip AB is put under tension by the aryvocalis fibers. These fibers are of microorder so their lateral and dorsal pull is just enough to make the segment receiving energy more tense than any segment not momentarily receiving such a stimulus. Segment CD is simultaneously put under tension in the same manner. But segment $B C$ receives no energy and is thus left relaxed. The pneumatic pressure from below strikes the glottic edges. Since segments $\mathrm{AB}$ and $\mathrm{CD}$ are tensed they will resist this blast but segment $\mathrm{BC}$ will allow the air to pass because of its 
laxity. This segment $\mathrm{BC}$ will immediately close again when the pressure is diminished to the extent that it is equal to the force of the elastic recoil of the lips, where they are parted. Repetition of this act will be periodic, which will initiate tone. Such tone may then be amplified by other mechanisms. If the pitch is to be altered to a higher tone, then some aryvocalis fibers inserted into segment $\mathrm{BC}$ continuous with $\mathrm{AB}$ at one end of the vocal lip and with CD at the other end, will be innervated. This will result in segment $\mathrm{BC}$, the temporary air valve becoming shorter. With a constant pressure of air this shorter segment will vibrate more rapidly and the tone will rise. In case the pitch is to be lowered, then some of the aryvocalis fibers of the segments $A B$ and $C D$, which at the moment are being innervated adjacent to segment $\mathrm{BC}$, will be denervated so that the relaxed segment $\mathrm{BC}$ will be longer. Other factors being constant the vibration rate will be slower and the tone will be lowered. Fxactness in change of pitch is attained because the hearing apparatus permits an instantaneous check on the rate of vibration emitted. Thus, almost infinite modulation in pitch is instantly possible. For this reason one learns to sing so easily. And correlated with this fact may be the reason why one remembers sound sequence more accurately than anything else, barring a musically defective ear.

The above-described mechanism we conceive to be the prime one for the production of pitch but not the exclusive one. The cricothyroid muscle, by its action in separating the thyroid and cricoid cartilages dorsoventrally, separates the arytenoid from the cricoid cartilage as well, for the arytenoids are attached to the cricoid. It therefore lengthens the upper condensed edge of the conus elasticus which is the vocal ligament. The thyroarytenoid muscle by its action shortens this ligament. Thus the glottic edge may be made longer or shorter over its whole length. Observation shows that the glottic edges may vibrate when the edges are not in contact to form the customary oval in the approximate center of the vocal lip. The edges are by structure always in contact ventrally, but 
diverge dorsally, and this dorsal part may vibrate in the lower tones. The cavity of the larynx below the glottic edges narrows so abruptly toward the glottis that the air blast from the trachea is greatly restricted. Therefore the glottic edges which project medially above this cavity afford impedence to the upward rush of air even though they lack complete contact dorsally. The force of air is resisted by the vocal ligaments whose elasticity allows them to be stretched and to recoil alternately, thereby leading to periodic motion which initiates sound. The aryvocalis fibers act to stiffen a definite length of the vibrating edges of the vocal lips, by their lateral tension, and thus determine the pitch. In the highest notes produced the vibrating mechanism must be, of necessity, very tenuous. Such vibrations, in singing, of the order of 1024 or 2048 cycles require a vibrating structure of extreme delicacy. It would, therefore, seem probable that very little of the vocal ligament is held between the layers of the vocal fold during vibration, in making such notes, but that even the fold itself may be spun out into a reduplicated edge like that illustrated in figure 2. In this case the conus and the vocal ligament would act as a framework over which the vocal fold could be set at a definite length to vibrate at a very high frequency. The vocal folds are continuously flooded above with beads of mucus at all times during phonation, so that the reduplicated edges of the vocal fold can well be spun out as figured. It is even possible that the mucus itself may form a thin extension of the glottic edge, capable of vibration in such pitches as are attained in the production of sibilants which are around 8000 cycles per second.

In a private communication to the writer, G. Oscar Russell, reporting his own observations of the epiglottic tubercle approaching the cuneiform tubercles, states that the passage above the ventricular folds is narrowed to a distance of 2 or $3 \mathrm{~mm}$. in diameter, during very slow vibrations. This restriction he claims impedes the vibrations in passing this barrier so that the vibration rate is mechanically lowered still more than the rate given the air by the vocal lip. It is in this way that he accounts for the production of very lowest tones. 
Certain evidence may be adduced from pathologic changes in the vocal apparatus which indicate the plausibility of the interpretation of pitch production here given. It is a wellknown fact that those born deaf never learn to carry a tune. This inability may be attributed to the fact that they cannot hear their own tones, so that the spiral organ cannot act as a modulating stimulus to certain central nervous connections which lead to speeific groups of neurones which innervate the different segments of the aryvocalis muscle fibers. Hence a monotone results. For the same reason otoselerotics usually speak high in pitch and loudly; they believe themselves to be speaking in an ordinary tone. In this case the auditory ossicles fail of normal function and in consequence the acoustic insult received by the tympanic membrane dissipates its frequency as well as its intensity in traversing the fused ossicles, and arrives at the oval window somewhat diminished. Hence the inner ear receives a diminished stimulus, and its possessor judges the sound emitted by his voice to be too low in pitch. To raise this to what he considers normal he innervates the aryvocalis fibers specifically to compensate for this lowness of pitch. In the case of edema involving the vocal fold and the vocal ligament, as in laryngitis or in the case of excessive cheering (yelling), there is a loss of ability to produce true pitch. Here again, a passive mechanism is indicated, for the nervous and muscular structures are intact, but the vocal ligament which is fascial and which has become edematous is involved. Since this ligament is passively vibrated the definite length and weight of the vibrating structure can no longer be set readily by neuromuscular control. Phenomena exhibited in vocal control, in cases of myxedema, likewise are attributable to a passively vibrated mechanism. Again when the rapid growth of the larynx, incident to puberty takes place, an upset of the control of pitch ensues. Stabilization is reached only after growth has ceased because specificity of innervation has been destroyed temporarily. In old age, which is accompanied by general lack of tonus of the stratified squamous epithelium of the whole body, the vocal folds show 
this condition by aiding in producing an enfeebled pitch control, which results in a quavery tone. On the other hand, when the general energy is low, the voice may be weak but the control of pitch is accurate. As age advances the elastic connective tissue becomes less elastic throughout the body, and, therefore, the vocal ligament is incapable of being spun out into as fine a wedge as needed for producing the highest notes. Correlated with this fact is the change of the vocal register from tenor to baritone in the male, and from soprano to mezzosoprano or alto in the female. The voice always falls in pitch indicating that the highest notes can no longer be made with ease or at all. There is an ability to use the lower notes better and new low notes, in addition, are added to the register.

All previous investigators of the larynx have affirmed that dorsoventral tension of the vocal ligament was the controlling factor of pitch production, in analogy to increased tension on a string of a stringed instrument. In altering the pitch of a string, by tension, one hears a chromatic blur or glissando effect, as the intervals are quickly passed over. Such is not the case in the human voice. Here each note stands out clearly stopped from the next. Linear tension of the vocal ligament then could not possibly have the result of isolating each note nor of avoiding glissando. Specific innervation of segments of the aryvocalis muscle fibers, in the manner maintained herein, however would accomplish this effect.

The analysis of the vocal mechanism here presented permits conclusions which account for the phenomena of pitch production as seen in the living larynx. Pitch is controlled by means of varying the length of the vibrating part of the vocal lip. This is accomplished chiefly by means of the aryvocalis muscle, which is so inserted into the vocal ligament as to eause the vibrating mechanism to vary its length at will. This vibrating mechanism is a valve, at times open, at times closed depending on the type of tone produced, and it is formed by the opposed glottic edges. This valve is made to vibrate passively by the pneumatic assault from the lungs, and it varies inversely, in length, as the height of the pitch to be attained. The thesis 
here defended violates no phenomena displayed in the living singing larynx, nor fact of relationships of the structures which initiate sound and which control pitch.

\section{LITERATURE CITED}

Ferrets, Antonits 1741 De la formation de la voix de lhomme. Memoires Acad. Roy. Sc. Paris., pp. 409-432.

Jacobson, Amexander 1887 Zur Lehre vom Ban u. Function d. Muscul. thyreoaryten. beim Menschen. Arch. f. Mikr. Anat., Bd. 29, S. 617.

Muckex, Floxd S. 1915 The natural method of voice production. Chas. Seribners' Sons. New York. Pp. 45-55.

MüLler, Johannes 1839 Elements of physiology. English translation by William Baly. Taylor \& Walton. London 1842. Vol. 2, pp. 1002-1036.

Negus, V. E. 1929 The mechanism of the larynx, G. V. Mosby Co. St. Louis. Pp. $422-439$.

Russell, G. Oscar 1931 Speech and Voice. The Macmillan Co. New York. 1932 Personal communication dated Norember 7 th. 